\title{
HMGB1, NLRP3, IL-6 and ACE2 Levels Are Elevated in COVID-19 with Headache: A Window to the Infection-Related Headache Mechanism
}

Hayrunnisa Bolay ( $\nabla$ hbolay@gazi.edu.tr)

Gazi University Faculty of Medicine https://orcid.org/0000-0002-3357-7733

Ömer Karadaş

University Health Science

Bilgin Öztürk

University of Health Sciences

Rıza Sonkaya

University of Health Sciences

Bahar Taşdelen

Mersin University

\section{Tuba DS Bulut}

Gazi University Faculty of Medicine: Gazi Universitesi Tip Fakultesi

Özlem Gülbahar

Gazi University Faculty of Medicine: Gazi Universitesi Tip Fakultesi

Aynur Özge

Mersin University Faculty of Medicine: Mersin Universitesi Tip Fakultesi

Betül Baykan

Istanbul University: Istanbul Universitesi

Research article

Keywords: COVID-19, headache, inflammation, NLRP3, HMGB1, ACE2, Angiotensin II, IL-6, IL-10, CGRP

Posted Date: July 9th, 2021

DOl: https://doi.org/10.21203/rs.3.rs-668048/v1

License: (1) (i) This work is licensed under a Creative Commons Attribution 4.0 International License.

Read Full License

Version of Record: A version of this preprint was published at The Journal of Headache and Pain on August 12th, 2021. See the published version at https://doi.org/10.1186/s10194-021-01306-7. 


\section{Abstract}

BACKGROUND AND AIM: Pathogenesis of COVID-19 -related headache is unknown, though the induction ofthe trigeminal neurons throughinflammation is proposed. Weaimed to investigatekey systemic circulating inflammatory moleculesand their clinical relations in COVID-19 patients with headache.

METHODS: Thiscross-sectional study enrolled 88COVID-19 patients, hospitalized on a regular ward during the second wave of the pandemic.Clinical characteristics of COVID-19 patients were recorded,and laboratory testswere studied.

RESULTS: The mean agesof 48 COVID-19 patients with headache (47.71 \pm 10.8$)$ and 40 COVID-19 patients without headache $(45.70 \pm 12.72)$ were comparable. COVID-19 patients suffered from headache had significantly higher serum levels of HMGB1, NLRP3, ACE2, and IL-6 than COVID-19 patients without headache, whereasCGRP and IL-10 levels were similar in the groups. Angiotensin II level was significantly decreased in the headache group.COVID-19 patients with headache showedan increased frequency of pulmonary involvement and increased D- dimer levels. Furthermore, COVID-19 was more frequently associated with weight loss, nausea, and diarrhea in patients with headache. The frequency of anosmia and ageusia did not reach significant levelsbetween the two groups. Serum NLRP3 levels were correlated with headache duration and hospital stay, while headache response to paracetamol was negatively correlated with HMGB1 and positively associated with IL-10 levels.

CONCLUSION: Stronger inflammatory response is associated with headache in hospitalized COVID-19 patients with moderate disease severity.Increased levels of the circulating inflammatory and/or nociceptive molecules like HMGB1, NLRP3, and IL-6 may play a role in the potential induction of the trigeminalsystem and manifestation of headache secondary to SARS-CoV-2 infection.

\section{Article Highlights:}

- Pathogenesis of viral systemic infection-related headache is unknown, though the inflammationmediated trigeminal activation was proposed for headache development in COVID-19.

- COVID-19 patients with headache had stronger inflammatory response and significantly elevated serum levels of HMGB1, NLRP3, ACE2, and IL-6 than COVID-19 patients without headache.

- Serum NLRP3 levels were correlated with headache duration and hospital stay, while headache response to paracetamol was negatively correlated with HMGB1 levels.

- Elevated levels of the circulating inflammatory and/or nociceptive molecules like HMGB1, NLRP3, and IL-6 may play a role in the potential induction of the trigeminal neurons and manifestation of headache secondary to SARS-CoV-2 infection.

\section{Introduction:}


COVID-19 is characterized by robust systemic inflammation and the release of inflammatory molecules and pro-inflammatory cytokines $(1,2)$. Headache is one of the presenting and frequent symptoms of COVID-19 (3-7). It is intriguing to note that headache is not seen in all COVID-19 patients or not only prevalent in those primary headache sufferers, such as migraine. Headache frequency was reported to be $12 \%$ in a meta-analysis (3) and $29 \%$ in hospitalized COVID 19 patients with moderate disease severity (5). New-onset, unrelenting, moderate to severe, frontally located, pulsating or pressing headaches, associated with the anosmia/ageusia and gastrointestinal complaints, especially when seen in men, were more frequently associated with COVID-19 (4-8).

The probable pathophysiology of the headache secondary to COVID-19 was suggested by us to involve inflammation, vasculopathy, or direct involvement of the trigeminal nerve in the upper respiratory system $(2,9)$. Activation of inflammasome complex by a direct SARS-CoV-2 protein induction and/or angiotensin II-mediated pathway was hypothesized to take a key role in the necessary neuroinflammation in the trigeminal system $(2,9)$. A recent prospective study demonstrated the involvement of inflammation in COVID-19 headache and disclosed two classes of headache phenotypes determined by several factors, including elevated circulating IL-6 levels (5). It has been reported that SARS-CoV-2 can directly activate NLRP3 (Nod-like receptor pyrin domain-containing 3), and different responses of COVID-19 patients could be attributed to differences in properly downregulation of NLRP3 inflammasome activation (10). Recent studies have also highlighted that the high mobility group box 1 (HMGB1) is one of the important host factors for COVID-19 pathogenesis. Regarding disease severity of COVID-19, a correlation with increased inflammatory responses is well-established and HMGB1 is a critical extracellular mediator in these inflammatory processes (11).

The potential nociceptive role of the elevated circulating pro-inflammatory cytokines because of COVID19 could play a role in the emergence of headache. The present study was aimed to investigate whether serum levels of NLRP3, HMGB1, IL-6, IL-10, angiotensin II and ACE2 were different in COVID-19 patients with headache. We also aimed to determine the relation of the above-mentioned inflammatory mediators and clinical features of headache in patients diagnosed with COVID-19.

\section{Methods:}

Subjects: COVID-19 patients $(n=107)$ hospitalized with moderate symptoms in the routine COVID-19 ward were consecutively recruited during the second wave of the pandemic in January and February 2021 for this cross-sectional study.

Inclusion criteria were COVID-19 diagnosis according to WHO interim guidance, polymerase chain reaction (PCR) positivity for SARS-CoV2, adults aged between 18-70 years, without any history of malignancy, severe metabolic syndrome, uncontrolled hypertension, uncontrolled diabetes, chronic liver failure, chronic respiratory diseases such as chronic obstructive pulmonary disease (COPD) or interstitial pulmonary disease, chronic renal failure, decompensated congestive heart failure, current alcohol/drug abuse, and major psychiatric disorders. Presence of headache was required for headache group. 
Exclusion criteria were patients transferred to ICU, headache attributed to other secondary causes, patients who had severe hearing or speech impairment to interfere with communication and patients who refused further laboratory investigations. COVID-19 patients with mild- moderate headaches, displaying VAS $\leq 7$ (visual analog scale) were excluded, to create reliably differentiating groups with versus without headache based on a previous report (5). Consequently, among 107 patients diagnosed with COVID-19 illness; 88 patients were enrolled in the study. Patients, who required mechanical ventilator or ICU stay within the first 10 days of hospitalization $(n=3)$, those diagnosed with other secondary headache etiologies $(n=3)$, and finally patients with lower VAS $\leq 7$ scores $(n=12)$ were excluded from the study.

The study was performed in accordance with the principles of the Helsinki Declaration and approved by the Local Ethical Committee and Ministry of Health Ethical Committee (Protocol No: 2021-18 and 202109T07-52-03 respectively). Written informed consent was obtained from all patients about the recruitment for scientific purposes. All patients with COVID-19 were diagnosed and managed with the same treatment protocol, approved by the Ministry of Health, consistent with WHO Guidelines (12).

Neurologists and headache specialists examined all COVID-19 patients and questioned for the absence/presence of headache and past headache history. Headache intensity was determined by VAS (VAS > 7: severe intensity). Other symptoms like anosmia, ageusia, nausea, weight loss and loss of appetite were recorded.

Nasopharyngeal and nasal swab specimens were studied for SARS-CoV-2 real-time reverse-transcription polymerase chain reaction (rRT-PCR) test. Thorax CT and routine laboratory tests (complete blood cell count, blood chemical analysis) were investigated in a standardized manner in all patients. D-dimer test has been performed by using a fluorescence immunoassay for the quantitative determination of crosslinked fibrin degradation products containing D-dimer in EDTA anticoagulated plasma specimens (reference values $<0.50 \mathrm{mcg} / \mathrm{mL}$ ).

To study inflammatory molecules, peripheral blood sample was taken from the patients at hospital admission. Serum samples were stored at -80 degrees until analyzed. Serum NLRP-3, IL-6, IL-10, HMGB-1, ACE2, Ang-II and CGRP levels were measured by ELISA (Enzyme-Linked Immuno Sorbent Assay) method using commercial kits according to the manufacturer's instructions. Human ELISA kits were obtained from Bioassay Technology Laboratory Shanghai Korain Biotech Co. (NLRP-3), Elabscience Biotechnology Inc. (IL-6, IL-10, HMGB-1, ACE2, Ang-II) and ELK Biotechnology (CGRP). Intra-assay and inter-assay CV values of methods were $<10 \%$. Researchers conducted laboratory tests and data analyst were blinded to the allocation of the patients.

Statistical analysis: Continuous data were summarized as mean \pm standard deviation and median (Q1Q3) values and categorical data were described with count (\%) values. Shapiro Wilk's test was used to check normality. The comparisons of COVID-19 patients with and without headache were made using independent samples $t$ test (mean age, HMGB1) or Mann Whitney U test (median NLRP3, IL-6, IL-10, angiotensin II, ACE-2, D-dimer). The relationship between categorical variables was evaluated by means of chi-square test and Fisher's exact test. To control familywise error rate in these comparisons about 
main hypothesis of study, Bonferroni adjustment was applied. In addition, Receiver operating characteristics (ROC) curves were used to obtain cut-off values associated with COVID-19 headache. It is simply based on the density estimation of variables that are observed for the case concerned. Pairwise comparison of ROC curves for cytokines were done using Delong's method. In the study, STATISTICA 13.0 (TIBCO Software Inc.) and MedCalc 19.8 (free trial version) softwares were used and statistical significance level was $p<0.05(13,14)$. The networks analysis, a relatively new and promising method for modeling interrelationships between large numbers of variables were performed by using the JASP software version $0.14(15)$.

\section{Results:}

Demographic features, clinical characteristics, and laboratory results of the two groups with and without headache were given in Table 1. Male dominance was notable in COVID-19 patients with headache; age distribution was similar in both groups. All patients received the same standard treatment protocol including low molecular weight heparin according to the regulations of Ministry of Health.

The presence of severe headache was significantly associated with nausea, loss of appetite, and loss of weight $(p<0.001)$ compared to COVID-19 patients without headache. The frequency of history of primary headache was not statistically different between the two groups. Pulmonary involvement was detected in 44 patients with COVID-19 and among them, 31 had headache with VAS $>7$. None of the included COVID19 patients suffered from hypoxia or required ventilation or had any serious complication including coagulopathy problem during their stay in the hospital. Hospitalization was about 2 days longer in COVID-19 patients with headache compared to non-headache sufferers (Table-1). Likewise, hospitalization days were less in COVID-19 patients without pulmonary infiltration compared to that of with pulmonary involvement ( $7.8 \pm 2.4$ vs $6.8 \pm 2.08 p=0.03)$. Headache was bilateral, severe, pulsating/pressing quality and unrelenting in COVID-19 patients that was consistent with our previous reports in other series $(4,5,8)$. COVID-19 related headache was unresponsive to paracetamol in $75 \%$ of the patients and its duration was $5.1 \pm 1.2$ days (median:5, range $4-8$ days).

COVID-19 patients with severe headache showed a higher ratio of pulmonary involvement diagnosed by thorax CT imaging compared to COVID-19 patients without headache $(65 \%$ vs $33 \%, p<0.001)$ (Table- 1 ). In terms of routine laboratory parameters, D-dimer levels were significantly higher in COVID-19 patients with severe headache (median: 6.20; 4.80-8.80) compared to COVID-19 patients without headache (median: $0.75 ; 0.20-1.95, p<0.001)$. COVID-19 patients with pulmonary involvement $(n=44)$ exhibited more frequent loss of appetite ( $n=26$ vs $n=10, p=0.001)$, loss of weight $(n=21$ vs $n=8, p=0.006)$ and nausea ( $n=20$ vs $n=6, p=0.002$ ). Symptoms of anosmia, ageusia, and diarrhea were not different in COVID-19 patients with pulmonary involvement compared to COVID-19 patients without pulmonary involvement.

Compared to COVID - 19 patients without headache, serum levels of HMGB1, NLRP3, IL-6, angiotensin II, and ACE2 were significantly higher in COVID-19 patients with severe headache (Figure-1, 2). Serum CGRP 
levels were not statistically significant between COVID-19 without headache (median:40.23 (21.1363.42) and with headache (median: $36.37(25.02-86.59, p=0.456)$. Serum IL-10 levels were not different between the two groups (median 13.01 (9.54-18.79) vs (median: $13.30(10.98-20.24), p=0.157)$.

COVID-19 patients with headache and pulmonary infiltration $(n=31)$ had significantly higher serum levels of HMGB1, IL-6, D-dimer (Table-2). NLRP3 was correlated with headache duration $(w=0.69)$ and hospital stay $(w=0.63)$ in COVID-19 patients with headache (Fig. 3). Response of headache to paracetamol 1000 mg was significantly lower in patients with pulmonary involvement ( $13 \%$ vs $47 \%, p=0.008)$. In COVID-19 headache patients who are unresponsive to paracetamol, HMGB1 level was significantly elevated (989.3 \pm 263.5 vs $514.7 \pm 248.9, p<0.001)$ whereas IL-10 level $(16.1 \pm 11.9$ vs $19.4 \pm 5.6 p=0.017)$ was decreased.

ROC curves for inflammatory markers indicated the significant role of HMGB1, NLRP3, IL-6 and ACE2 in discriminating headache in COVID-19 (Figure-2). Network plot analyses illustrated the positive and negative relationship among inflammatory molecules and COVID-19 headache (Fig. 3A), headache duration, hospital stay and paracetamol response (Fig. 3B).

\section{Discussion:}

SARS-CoV-2 primarily causes a respiratory tract infection and is associated with increased circulating proinflammatory cytokines and inflammatory mediators, with a potential progression to cytokine storm $(1,16)$. Though assumed by Bolay et al $(2,9)$, it had not been shown so far whether inflammatory molecules were associated with COVID-19 headache. We studied peripheral circulating inflammatory molecules in COVID-19 patients with moderate severity hospitalized in the routine ward. Presence of severe headache in COVID-19 was associated with elevated inflammatory and/or nociceptive molecules like HMGB1, NLRP3, IL-6, and ACE2 compared to the non-headache COVID-19 sufferers, while antiinflammatory cytokine levels of IL-10 did not show any difference at hospital admission between the two groups. Moreover, NLRP3 and HMGB1 were correlated with headache duration and paracetamol unresponsiveness respectively. Robust inflammatory response was detected particularly in COVID-19 patients with severe headache. In supporting, these patients were also associated with increased frequency of pulmonary involvement but not needing any ICU management.

Angiotensin-converting enzyme 2 (ACE2) has gained importance in COVID-19 pathogenesis because membrane-bound ACE2 is the receptor for SARS-CoV-2 cellular entry $(2,9,17)$. ACE2 is widely expressed in the human body, including epithelial cells in nasal and oral mucosa, pneumocytes in the respiratory system, besides the vascular endothelial cells and smooth muscle cells (18). Soluble ACE2 on the other side could reflect the cleavage of membrane-bound ACE2 by the SARS-CoV-2 entry and lysis of ACE2expressing cells. High levels of plasma ACE2 was proposed to indicate poor outcome in critically ill patients $(19,20)$. The moderately affected disease process was different in our patients, in whom elevated ACE2 levels were seen in association with good outcomes without any ARDS, serious 
complication or mortality. Increased circulating ACE2 level was even suggested to denote endogenous protection against SARS-CoV-2 $(21,22)$.

Angiotensin II levels detected in our study merit particular emphasis. Unless cleaved by ACE2 enzyme, angiotensin II peptide mediates various functions including regulation of blood pressure, vascular tonus, electrolyte balance, and induce inflammation, produce reactive oxygen species and activate NLRP3 inflammasome $(2,9,17,23,24)$. The internalization of membrane bound ACE2 by the virus binding would result in the unbalanced activity of angiotensin II, yielding NLRP3 inflammasome activation and release of pro-inflammatory cytokines subsequently recruiting inflammatory cells $(2,9,18,23)$. Angiotensin II is also involved in pain signaling in the trigeminal ganglia (25) and in inducing nociceptive behavior (26). Besides, angiotensin II receptor inhibitors are effective prophylaxis drugs for migraine headache (27) and implicated in neuropathic pain treatment (28). Consequently, elevated circulating angiotensin II levels in COVID-19 patients with severe headache were assumed to contribute to trigeminal nociception and indicate increased activity of inflammatory cascade via its receptors $(2,9)$. However, our study failed to show elevated serum levels of angiotensin II in COVID - 19 patients with headache. The circulating angiotensin II levels were significantly decreased in patients with COVID-19 headache on the contrary to elevated ACE2 levels. Increased circulating ACE2 may cleave angiotensin II and be responsible for the reduced angiotensin II at the point of our measurements in COVID-19 patients. Decreased serum angiotensin II peptide levels were already shown in COVID-19 patients (29). Taken all together, our results may suggest that decreased circulating angiotensin II is implausible to be responsible for the headache in COVID-19 patients. Likewise, comparable systemic circulating CGRP levels in COVID-19 patients with or without headache groups in our study lessens the role systemic CGRP as a potential trigger for COVID-19 related headache. That was in line with the recent study that reported lower serum CGRP levels in COVID19 patients (30).

Elevated HMGB1 level is significantly associated with the emergence of severe headache and correlated with paracetamol unresponsiveness in our patients with COVID-19 headache. HMGB1 is a prototypical damage-associated molecular pattern (DAMP) representing a critical marker of intense inflammation and studies have shown that serum HMGB1 is increased in COVID-19 cases and positively correlated with disease severity. Moreover, serum HMGB1 also decreases when patients improved (31, 32). HMGB1 may be involved in the cytokine storm or as a second way also by ACE2 expression in alveolar epithelial cells, in the COVID-19 pathogenesis. Increased levels of serum HMGB1 are considered a reliable marker for systemic inflammation but do not indicate the specific tool initially involved in the inflammatory disease. Additionally, HMGB1 regulates autophagy, which is related to SARS-CoV-2 entry. The complex role of HMGB1 in migraine headache was reviewed in detail (33). HMGB1 can cause nociception via direct activation of receptor for advanced glycation end-products (RAGE) in sensory neurons (34). HMGB1 is also implicated in maintaining the neuropathic pain state and trigeminal neuropathic pain. Treatment of the trigeminal nerve with anti-HMGB1 neutralizing antibody prevented pain behavior and blocked macrophage and microglia activation (35). Therefore, HMGB1 could be a key player in the development of severe and long-lasting COVID-19 headache. Our results indicated that it will be important to study the 
potential role of HMGB1 in COVID-19-related headache in related models to gain more insight into the headache mechanisms triggered by external sources, specifically COVID-19.

In COVID-19, SARS-CoV activates NLRP3 inflammasome, which is critical in the innate immune defense system, yielding proinflammatory cytokines including IL- 6 , prostaglandins, and leukotrienes to amplify tissue inflammation $(9,23,24,36)$. Recently, the possible role of NLRP3 inflammasome were implicated in the development of headache in COVID-19 (9). NLRP3 was significantly associated with headache in COVID-19 and correlated with duration of headache and hospital stay, in our study. In glyceryl trinitrateinduced experimental headache studies, activation of NFkb, IL1 $\beta$, IL-6 and NLRP3 inflammasome were shown in dural macrophages and in the brainstem microglia (37-39). However, the exact role of circulating NLRP3 levels in inducing trigeminal nociception needs to be determined by further studies.

Development of headache represents a nociceptive activation of the trigeminal nerve through the neuroinflammatory-vascular processes, where release of CGRP from perivascular trigeminal nerve endings, vascular reactivity and inflammation take place $(2,9,40)$. Pro-inflammatory cytokine IL- 6 was demonstrated to activate perivascular trigeminal nociceptors in the dura mater and induce headache in experimental studies $(41,42)$. Additionally, IL-6 was reported to provoke CGRP release particularly under heat conditions (43). Therefore, higher circulating levels of IL-6 in patients with more intense headache could point out a potential trigger role of IL-6 in COVID-19 headache.

Pulmonary involvement was detected by thorax CT in half of all patients and $65 \%$ of the COVID-19 headache sufferers. Intriguingly, D-dimer levels were significantly higher in patients with headache compared to non-headache sufferers. Increased serum D-dimer has been considered to reflect coagulopathy and poor prognosis in severe COVID-19 patients (44), but we observed no thrombotic event or mortality in our study group. In COVID-19 patients with moderate disease severity, higher levels of Ddimer and IL-6 with increased frequency of pulmonary infiltration were associated with severe headache (5). As proposed by Hunt \& Levi, D-dimer levels could represent the degree of lung inflammation, like other acute-phase proteins in COVID-19 (45). The latter would provide a rational explanation for the correlation of D-dimer elevation and pulmonary infiltration in our COVID-19 patients with headache. Moreover, the presence of headache in hospitalized COVID-19 patients was stated as an independent predictor of lower risk of mortality (46). Our results are different, as COVID-19 patients with hypoxia and who required ventilation in the ICU were not included in our study group. Yet, $35 \%$ of the patients without any pulmonary infiltration (Table-3) manifested severe headache intensity and elevation of IL-6, HMGB1. It seems that circulating inflammatory cytokines could also be associated with headache without pulmonary inflammation detected by CT.

We think that the headache correlated with the presence of pulmonary infiltration cannot merely be attributed to hypoxia as severe hypoxemia and/or hypercapnia were not identified in any of our patients. Rather, we propose pro-inflammatory mediators and cytokines released extremely during pulmonary infiltration could play a key role in the development of headache in the COVID-19 course. A recent study revealed that IL-6 levels were positively correlated with VAS scores in COVID-19 patients with headache 
(5). Also, ROC curve cut-off values were reported to indicate a moderate increase of IL-6 for defining COVID-19 headache (5). Both IL-6 and IL-10 are reported to be predictors of COVID-19 severity (47). The discordance of these cytokines, we found in COVID-19 patients with versus without headache, deserves special attention. IL-10, a typical anti-inflammatory cytokine did not show any difference in relation to the presence of headache, whereas IL-6, a major pro-inflammatory cytokine showed significantly higher levels in COVID-19 patients with headache, intriguingly. Even though IL- 6 and IL-10 were noted as predictors for COVID-19 deterioration, all of our patients were followed up in the hospital but not in ICU. The similarity of the IL-10 levels could be due to the early sampling at the hospital admission, its course would be different during the COVID-19 process.

Our clinical study has some constraints, as the study was conducted in hospitalized patients in the general ward, without including patients in the ICU and non-hospitalized mild symptomatic patients. In future studies, other groups with COVID-19 headaches like those with significant comorbidities, those transferred to the ICU, and subjects with mild/moderate (VAS $\leq 7$ ) headaches should be investigated prospectively to see the clinical relations and changes of the cytokines over time. Another potential limitation was that our results may indicate more severe involvement in cases with headache in comparison to those without and may reflect recruitment bias. A limited number of inflammatory molecules were studied at hospital admission and follow-up data providing their temporal progression during COVID-19 headache is lacking.

This pioneering study on headache in COVID-19 has several implications. So far, it is the first clinical study investigating the role of circulating inflammatory and nociceptive molecules such as HMGB1, NLRP3 inflammasome, IL-6, IL-10, angiotension II, and ACE2 in COVID-19 headache. Elevated HMGB1, NLRP3 and IL-6 levels could be discriminative for COVID-19 headache and may have implications for secondary headaches related to other systemic viral infections. No significant association of serum CGRP level with COVID-19 related headache is notable and rise the importance of HMGB1, NLRP3, IL-6 in COVID-19 headache pathogenesis. NLRP3 was correlated with headache duration and hospital stay, while paracetamol response was negatively associated with HMGB1 levels and positively correlated with IL-10 levels. Moreover, the current study conducted during a different wave of the pandemic confirmed the previous report that higher IL-6 levels and more frequent pulmonary infiltration were detected in COVID-19 patients with headache.

\section{Conclusion}

We conclude that significant elevation of nociceptive and inflammatory molecules like HMGB1, NLRP3, and IL- 6 could induce trigeminal activation leading to headache during the SARS-COV-2 infection. This significant participation of circulating inflammatory mediators in trigeminal nociception does not necessarily exclude the possible involvement of trigeminal nerve activation by SARS-CoV-2 directly or secondary to vasculopathy. In contrast to previous reports in severe cases, circulating higher levels of ACE2, HMGB1, IL-6 and D-dimer are not associated with poor outcome in COVID-19 patients with moderate disease severity and not necessitating ICU. 


\section{Declarations}

Ethics approval and consent to participate: All participants received a complete description of the study and granted written informed consentLocal Ethical Committee and Ministry of Health Ethical Committee (Protocol No: 2021-18 and 2021-09T07-52-03) were obtained.

\section{Consent for publication}

Not applicable.

\section{Competing interest}

The authors declare that they have no competing interests.

\section{Availability of data and materials}

The datasets generated during and/or analysed during the current study are not publicly available as the signed informed consent form by all patients did not include a provision stating that individual raw data can be made publicly accessible but are available from the corresponding author on reasonable request.

Funding: The authors did not receive funding for the design of the study and acquisition, analysis, and interpretation of data and in writing the manuscript.

Authors' contributions: $\mathrm{HB}, \mathrm{OK}, \mathrm{AO}$ and $\mathrm{BB}$ made substantial contributions to protocol development, interpretation of data as well as in drafting the manuscript. OK, BO, RS, OG, TO contributed to data acquisition and BT contributed data analysis and statistics. The author(s) read and approved the final manuscript.

\section{Corresponding author}

Correspondence to HayrunnisaBolay

Acknowledgements: TÜBA (Turkish Academy of Science) partially supported the research for this paper

\section{References}

1. Bohn MK, Hall A, Sepiashvili L, et al (2020) Pathophysiology of COVID-19: Mechanisms underlying disease severity and progression. Physiology (Bethesda) 35: 288-301.

2. Bolay H, Gül A, Baykan B (2020) COVID-19 is a real headache! Headache 60: 1415-1421.

3. Fu L, Wang B, Yuan T, et al (2020) Clinical characteristics of coronavirus disease 2019 (COVID-19) in China: A systematic review and meta-analysis. J Infect 80; 656-665. 
4. Uygun Ö, Ertaş M, Ekizoğlu E, et al (2020) Headache characteristics in COVID-19 pandemic-a survey study. J Headache Pain 21: 121.

5. Karadaş Ö, Öztürk B, Sonkaya AR, et al (2021) Latent class cluster analysis identified hidden headache phenotypes in COVID-19: impact of pulmonary infiltration and IL-6. Neurol Sci42 :16651673.

6. Toptan T, Aktan Ç, Başarı A, Bolay H (2020) Case series of headache characteristics in COVID-19: Headache can be an isolated symptom. Headache 60: 1788-1792.

7. Porta-Etessam J, Matías-Guiu JA, González-García N, et al (2020) Spectrum of headaches associated with SARS-CoV-2 infection: Study of healthcare professionals. Headache 60: 1697-1704.

8. Baykan B, Özge A, Ertaş M, et al(2021) Urgent need for ICHD Criteria for COVID-19-related headache: Scrutinized classification opens the way for research. Noro Psikiyatr Ars 58: 79-80.

9. Bolay H, Özge A, Uludüz D, Baykan B(2020) Are migraine patients at increased risk for symptomatic Covid-19 due to shared comorbidities? Headache 60: 2508-2521.

10. van den Berg DF, Te Velde AA (2020)Severe COVID-19: NLRP3 inflammasome dysregulated. Front Immuno/11: 1580.

11. Gowda P, Patrick S, Joshi SD, et al (2021) Glycyrrhizin prevents SARS-CoV-2 S1 and Orf3a induced high mobility group box 1 (HMGB1) release and inhibits viral replication. Cytokine 142: 155496.

12. World Health Organization (WHO) (accessed in 2020)Clinical management of severe acute respiratory infection when novel coronavirus ( $\mathrm{nCoV}$ ) infection is suspected, www.who.int/ publications-detail/clinical-management-of-severe-acute-respiratoryinfection-when-novel coronavirus- (ncov)-infection-is-suspected.

13. TIBCO Software Inc. Statistica (data analysis software system), version 13. http://tibco.com. (2018).

14. MedCalc $®$ Statistical Software version 19.8 (MedCalc Software Ltd, Ostend, Belgium; https://www.medcalc.org (2021).

15. Love J, Selker R, Marsman M, et al. (2019)JASP: Graphical statistical software for common statistical designs. J Stat Softw 88: 1-17.

16. Song P, Li W, Xie J, et al (2020) Cytokine storm induced by SARS-CoV-2. Clin Chim Acta 509: 280-287.

17. Zhang $\mathrm{H}$, Penninger JM, Li Y, et al(2020) Angiotensin-converting enzyme 2 (ACE2) as a SARS-CoV-2 receptor: Molecular mechanisms and potential therapeutic target. Intensive Care Med 46: 586-590.

18. Beyerstedt S, Casaro EB, Rangel ÉB(2021)COVID-19: angiotensin-converting enzyme 2 (ACE2) expression and tissue susceptibility to SARS-CoV-2 infection. Eur J Clin Microbiol Infect Dis 40: 905919.

19. Kragstrup TW, Singh, HS, Grundberg I. et al(2021)Plasma ACE2 levels predict outcome of COVID-19 in hospitalized patients. PLoS One. 16(6):e0252799.

20. Rahman MM, Hasan M, Ahmed A (2021) Potential detrimental role of soluble ACE2 in severe COVID19 comorbid patients. Rev Med Viro/10.1002/rmv.2213. Epub ahead of print. 
21. Ciaglia E, Vecchione C, Puca AA (2020) COVID-19 infection and circulating ACE2 levels: Protective role in women and children. Front Pediatr 23:206.

22. Nagy B Jr, Fejes Z, Szentkereszty Z, et al (2021) A dramatic rise in serum ACE2 activity in a critically ill COVID-19 patient. Int J Infect Dis 103: 412-414.

23. Shah $A(2020)$ Novel coronavirus-induced NLRP3 inflammasome activation: A potential drug target in the treatment of COVID-19. Front Immunol 11:1021.

24. Nieto-Torres JL, Verdiá-Báguena C, Jimenez-Guardeño JM, et al (2015)Severe acute respiratory syndrome coronavirus $\mathrm{E}$ protein transports calcium ions and activates the NLRP3 inflammasome. Virology 485:330-339.

25. Imboden H, Patil J, Nussberger J, et al (2009) Endogenous angiotensinergic system in neurons of rat and human trigeminal ganglia. RegulPept, 154: 23-31.

26. Nemoto W, Nakagawasai O, Yaoita F, et al (2013) Angiotensin II produces nociceptive behavior through spinal AT1 receptor-mediated p38 mitogen-activated protein kinase activation in mice. Mol Pain; 9: 38.

27. Tronvik E, Stovner LJ, Helde G, et al (2003)Prophylactic treatment of migraine with an angiotensin II receptor blocker: a randomized controlled trial. JAMA 2003; 289:65-69.

28. Rice ASC, Dworkin RH, McCarthy TD, et al (2014) EMA401, an orally administered highly selective angiotensin II type 2 receptor antagonist, as a novel treatment for postherpetic neuralgia: a randomised, double-blind, placebo-controlled phase 2 clinical trial. Lancet, 383:1637-1647.

29. Kutz A, Conen A, Gregoriano C, et al (2021) Renin-angiotensin-aldosterone system peptide profiles in patients with COVID-19. Eur J Endocrino/184: 543-552.

30. Ochoa-Callejero L, García-Sanmartín J, Villoslada-Blanco P (2021)Circulating Levels ofCalcitonin Gene-Related Peptide Are Lower in COVID-19 Patients. J EndocrSoc5(3):bvaa199.

31. Chen R, Huang Y, Quan J, et al(2020)HMGB1 as a potential biomarker and therapeutic target for severe COVID-19. Heliyon 6: e05672.

32. Chen L, Long X, Xu Q, et al(2020) Elevated serum levels of S100A8/A9 and HMGB1 at hospital admission are correlated with inferior clinical outcomes in COVID-19 patients. Cell Mol Immunol 17: 992-994.

33. Kursun O, Yemisci M, van den Maagdenberg AMJM, Karatas H. (2021). Migraine and neuroinflammation: the inflammasome perspective. $J$ Headache Pain.22(1):55.

34. Bestall SM, Hulse RP, Blackley Z, et al (2018) Sensory neuronal sensitisation occurs through HMGB-1RAGE and TRPV1 in high-glucose conditions. J Cell Sci; 131: jcs215939.

35. Kochi T, Nakamura Y, Ma S, et al (2021) Pretreatment with high mobility group box-1 monoclonal antibody prevents the onset of trigeminal neuropathy in mice with a distal infraorbital nerve chronic constriction injury. Molecules26(7):2035.

36. Rodrigues TS, de Sá KSG, Ishimoto AY, et al (2021) Inflammasomes are activated in response to SARS-CoV-2 infection and are associated with COVID-19 severity in patients. J Exp Med 218: 
e20201707.

37. Reuter U, Bolay H, Jansen-Olesen I, et al (2001) Delayed inflammation in rat meninges: implications for migraine pathophysiology. Brain;124: 2490-2502.

38. Reuter U, Chiarugi A, Bolay H, Moskowitz MA (2002) Nuclear factor-kappaB as a molecular target for migraine therapy. Ann Neurol 51:507-516.

39. He W, Long T, Pan Q, et al (2019) Microglial NLRP3 inflammasome activation mediates IL-1 $\beta$ release and contributes to central sensitization in a recurrent nitroglycerin-induced migraine model. $J$ Neuroinflammation16:78.

40. Ashina M, Hansen JM, Do TP, et al (2019) Migraine and the trigeminovascular system-40 years and counting. Lancet Neuro/18: 795-804.

41. Yan J, Melemedjian OK, Price TJ, et al (2012) Sensitization of dural afferents underlies migrainerelated behavior following meningeal application of interleukin-6 (IL-6). Mol Pain 8: 6.

42. Burgos-Vega CC, Quigley LD, Trevisan Dos Santos G, et al (2019) Non-invasive dural stimulation in mice: A novel preclinical model of migraine. Cephalalgia 39: 123-134.

43. Oprée A, Kress M (2000) Involvement of the proinflammatory cytokines tumor necrosis factor-alpha, IL-1 beta, and IL-6 but not IL-8 in the development of heat hyperalgesia: effects on heat-evoked calcitonin gene-related peptide release from rat skin. J Neurosci 20: 6289-6293.

44. He X, Yao F, Chen J, et al(2021) The poor prognosis and influencing factors of high D-dimer levels for COVID-19 patients. Sci Rep 11:1830.

45. Hunt BJ, Levi M. (2020). The source of elevated plasma D-dimer levels in COVID-19 infection. Br J Haematol; 190:e133-e134.

46. Trigo J, García-Azorín D, Planchuelo-Gómez Á, et al (2020) Factors associated with the presence of headache in hospitalized COVID-19 patients and impact on prognosis: a retrospective cohort study. $J$ Headache Pain 21:94.

47. Han H, Ma Q, Li C, et al (2020)Profiling serum cytokines in COVID-19 patients reveals IL-6 and IL-10 are disease severity predictors. Emerg Microbes Infect 9: 1123-1130.

\section{Tables}

Table-1:Demographic features, clinical characteristics, and laboratory results of the two groups with and without headache. 


\begin{tabular}{|llll|}
\hline & $\begin{array}{l}\text { COVID-19 } \\
\text { Without Headache } \\
\text { n=40 }\end{array}$ & $\begin{array}{l}\text { COVID-19 } \\
\text { With Headache }\end{array}$ & \\
\hline Mean Age (years) & $45.70 \pm 12.72$ & $47.71 \pm 10.78$ & 0.425 \\
\hline Women/ Men & $19 / 21$ & $16 / 32$ & 0.176 \\
\hline Primary headache & 3 & 11 & 0.077 \\
\hline Anosmia & 1 & 7 & 0.066 \\
\hline Ageusia & 1 & 5 & 0.214 \\
\hline Nausea & 3 & 23 & $<0.001$ \\
\hline Loss of appetite & 7 & 29 & $<0.001$ \\
\hline Weight loss & 4 & 25 & $<0.001$ \\
\hline Diarrhea & 7 & $8.38 \pm 2.42$ & $<0.001$ \\
\hline Pulmonary involvement & 13 & 31 & 0.216 \\
\hline D-dimer (mg/mL) & $1.22 \pm 1.18$ & & 0.003 \\
\hline Hospitalization (days) & $6.08 \pm 1.37$ & & $<0.001$ \\
\hline
\end{tabular}

Table-2: Comparison of the inflammatory molecule levels in COVID-19 patients with headache regarding pulmonary involvement. 


\begin{tabular}{|c|c|c|c|c|c|}
\hline & \multicolumn{5}{|c|}{ COVID-19 Patients with Headache $(n=48)$} \\
\hline & $\begin{array}{l}\text { Pulmonary Infilt } \\
(n=31) 65 \%\end{array}$ & & $\begin{array}{l}\text { Without Pulmor } \\
(n=17) 35 \%\end{array}$ & Iry Infiltration & \\
\hline & Mean $\pm S D$ & $\begin{array}{l}\text { Median } \\
\text { (Q1-Q3) }\end{array}$ & Mean \pm SD & $\begin{array}{l}\text { Median } \\
\text { (Q1-Q3) }\end{array}$ & $\begin{array}{l}\mathrm{P} \\
\text { value }\end{array}$ \\
\hline NLRP3 (ng/mL) & $3.03 \pm 3.92$ & $\begin{array}{l}1.43 \\
(1.15-3.32)\end{array}$ & $3.18 \pm 4.91$ & $\begin{array}{l}1.53 \\
(1.11-2.70)\end{array}$ & 0.982 \\
\hline IL-6 (pg/mL) & $141.63 \pm 126.2$ & $\begin{array}{l}100.09 \\
(26.04-217.05)\end{array}$ & $34.13 \pm 18.83$ & $\begin{array}{l}31.84 \\
(20.70-48.39)\end{array}$ & 0.036 \\
\hline IL-10 (pg/mL) & $18.12 \pm 12.46$ & $\begin{array}{l}13.58 \\
(10.98-20.24)\end{array}$ & $14.96 \pm 6.37$ & $\begin{array}{l}12.72 \\
(10.40-19.66)\end{array}$ & 0.283 \\
\hline Ang II (pg/mL) & $416.22 \pm 188.78$ & $\begin{array}{l}366.45 \\
(267.33-524.59)\end{array}$ & $471.62 \pm 165.93$ & $\begin{array}{l}443.07 \\
(401.79- \\
514.73)\end{array}$ & 0.111 \\
\hline ACE2 $(\mathrm{ng} / \mathrm{mL})$ & $11.65 \pm 5.40$ & $\begin{array}{l}10.71 \\
(7.36-15.72)\end{array}$ & $9.02 \pm 3.63$ & $\begin{array}{l}8.93 \\
(7.39-10.60)\end{array}$ & 0.073 \\
\hline $\begin{array}{l}\text { HMGB1 } \\
(\mathrm{pg} / \mathrm{mL})\end{array}$ & $960.17 \pm 318.71$ & $\begin{array}{l}964.87 \\
(724.42- \\
1205.49)\end{array}$ & $707.48 \pm 294.84$ & $\begin{array}{l}759.38 \\
(485.12- \\
923.55)\end{array}$ & 0.012 \\
\hline $\begin{array}{l}\text { D-dimer } \\
\text { (mg/mL) }\end{array}$ & $10.26 \pm 8.00$ & $\begin{array}{l}7.20 \\
(5.50-13.90)\end{array}$ & $5.05 \pm 2.82$ & $\begin{array}{l}4.90 \\
(2.90-6.80)\end{array}$ & 0.011 \\
\hline $\begin{array}{l}\text { CGRP } \\
(\mathrm{pg} / \mathrm{mL})\end{array}$ & $86.13 \pm 139.05$ & $\begin{array}{l}34.72 \\
(25.02-67.22)\end{array}$ & $208.48 \pm 290.35$ & $\begin{array}{l}55.46 \\
(20.92- \\
287.63)\end{array}$ & 0,321 \\
\hline
\end{tabular}

\section{Figures}



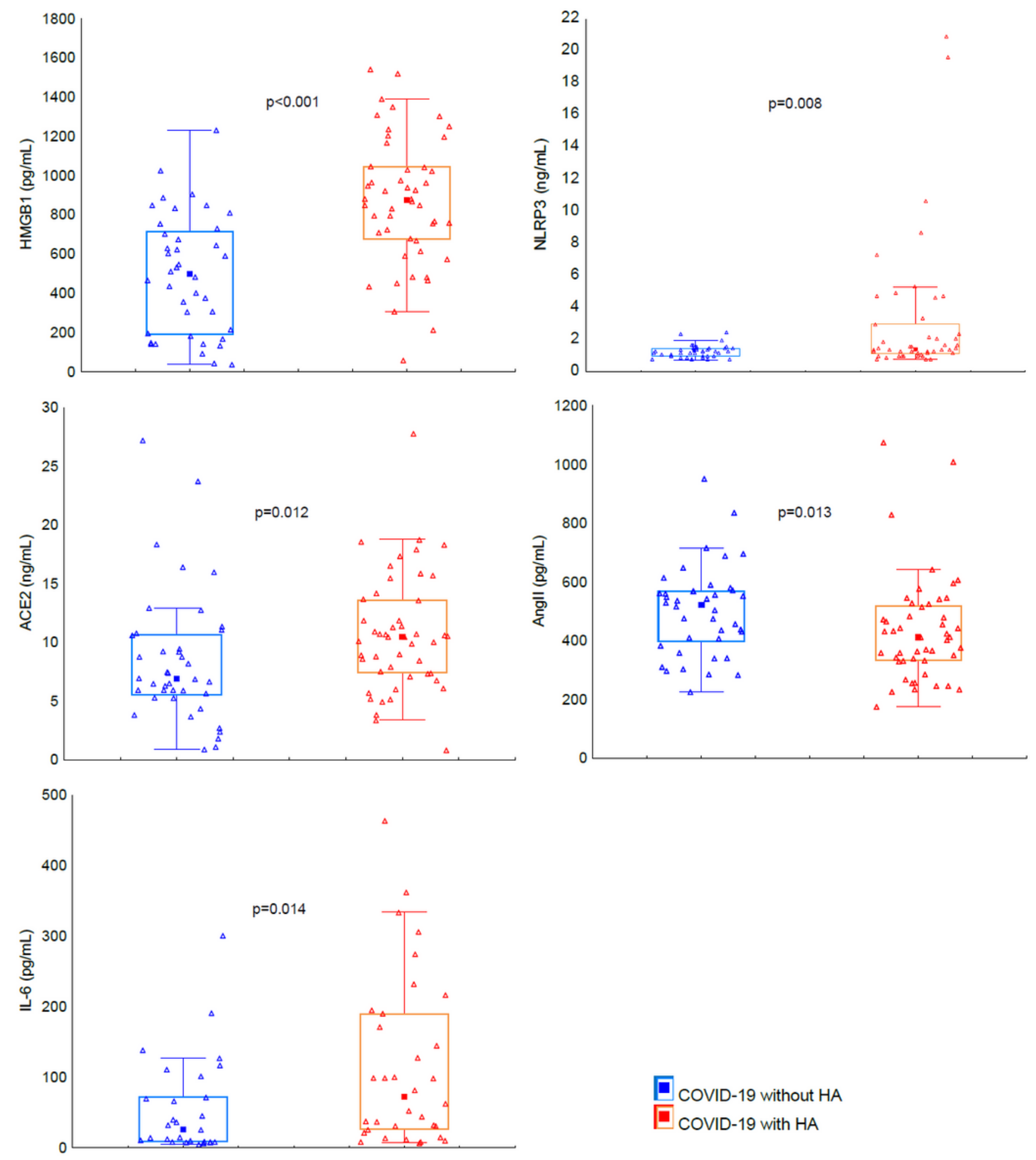

COVID-19 without HA

$\square$ COVID-19 with HA

\section{Figure 1}

Serum levels of inflammatory molecules at hospital admission in COVID-19 patients. HMGB1, NLRP3, ACE2, IL-6 were significantly increased in COVID-19 patients with headache compared to COVID-19 patients without headache. Angiotensin II level was significantly decreased in COVID-19 patients with headache. 


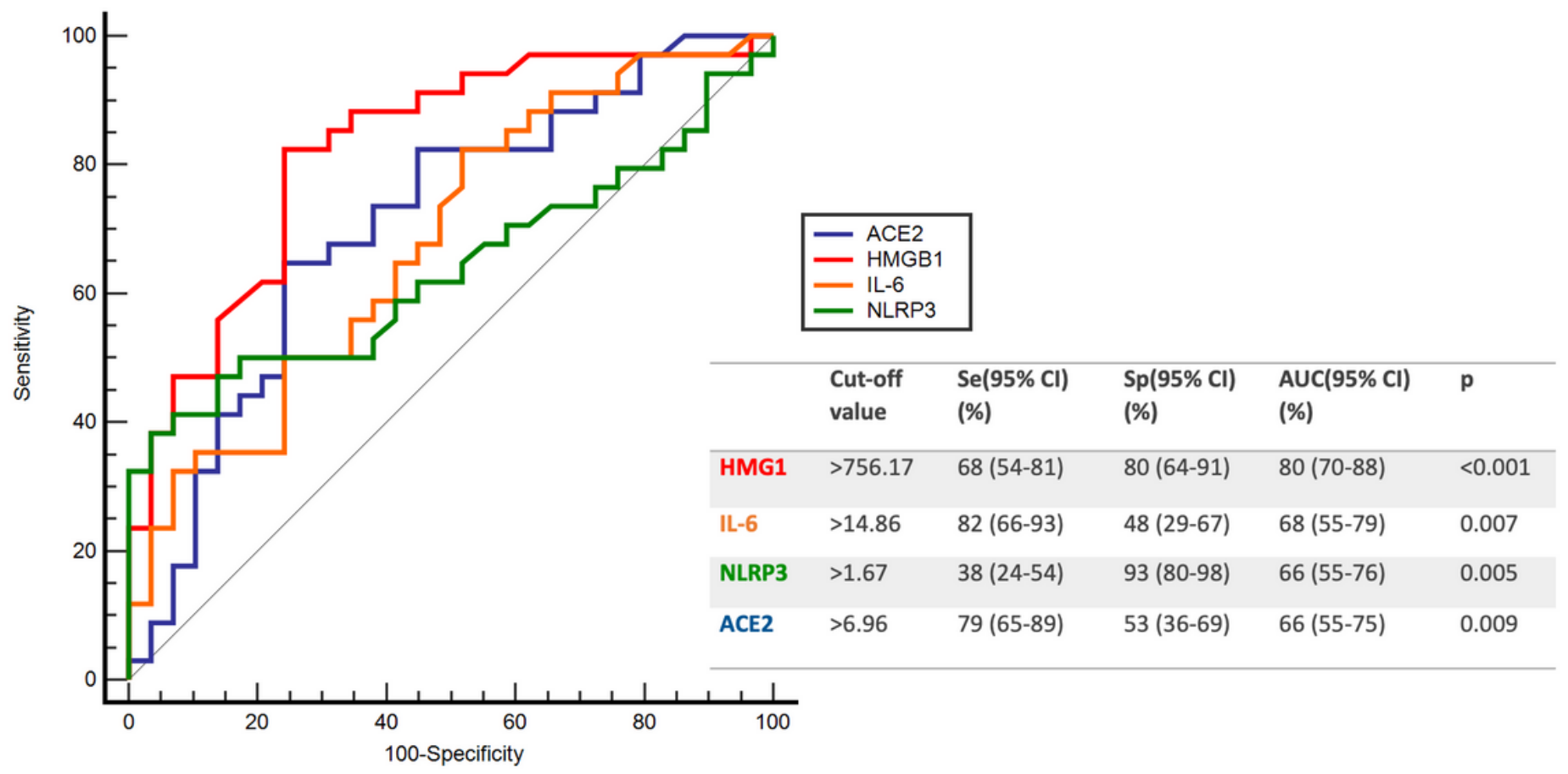

Figure 2

Independent ROC curves illustrated the diagnostic abilities of HMGB1, IL-6, NLRP3 and ACE2. Both sensitivity (Se) and specificity (Sp) values showed that classification performance of HMGB1 was better than the others. On the other hand, IL- 6 and ACE2 were found to be successful in classifying COVID-19 patients with headache, while NLRP3 were found to be more successful in classifying COVID-19 patients without headache. Combined performance of these inflammatory molecules was found to be highly successful (Area under curve AUC=0.92, $\mathrm{Se}=0.83, \mathrm{Sp}=0.88$ ). 


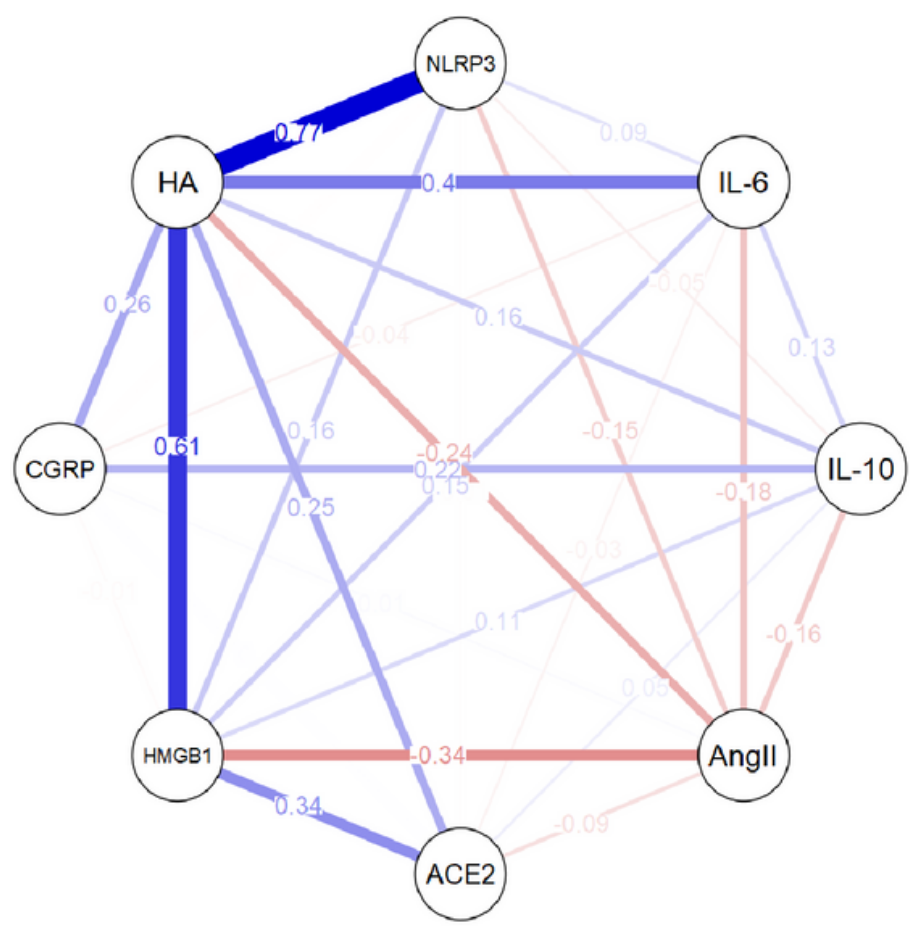

a

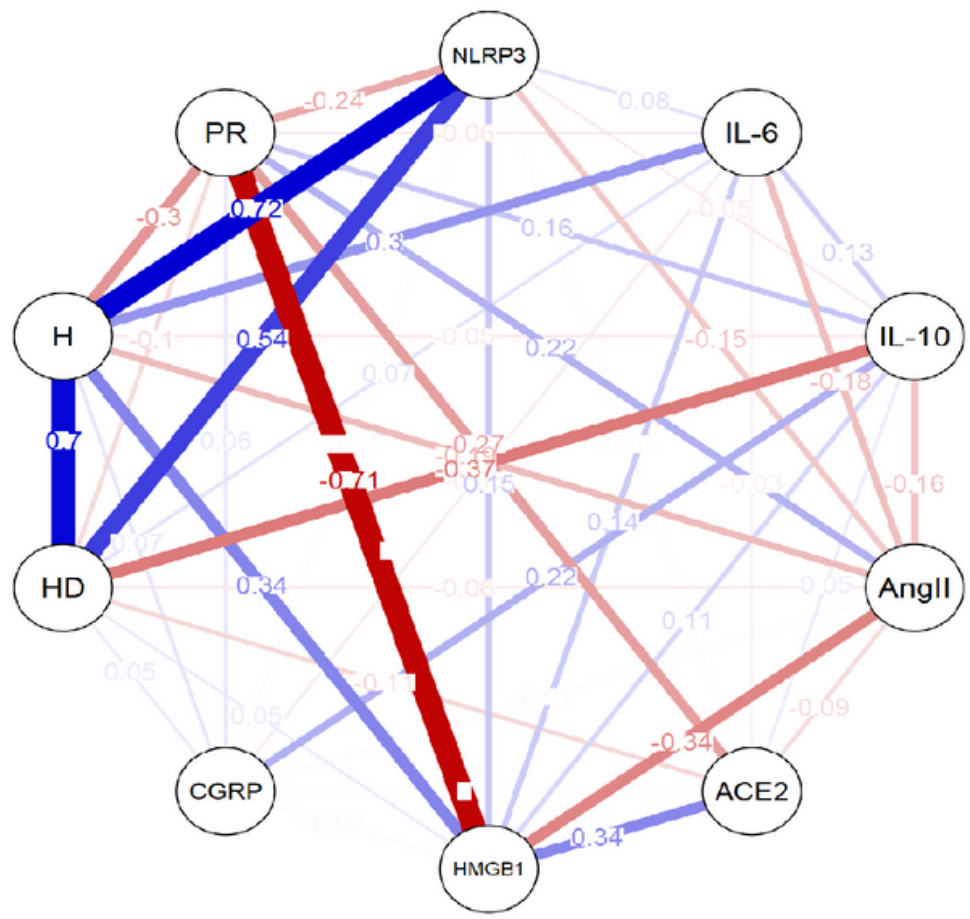

b

\section{Figure 3}

Network analysis models of the interrelationships among inflammatory molecules, headache (HA), headache duration (HD), hospitalization $(H)$ and paracetamol response (PR). Headache was positively correlated with serum levels of NLRP3, HMGB1, IL-6 and ACE2 and these relationships were greater than the others (A). Serum HMGB1 level was negatively correlated with paracetamol response $(w=-0.71)(B)$. Hospitalization $(w=0.72)$ and headache duration $(w=0.54)$ was positively correlated with NLRP3 level $(B)$. Line thickness denotes weight of the correlation, blue and red color represent positive and negative correlations, respectively. 